\title{
Learning with Smart Multipurpose Interactive Learning Environment
}

\author{
Mária Bieliková, Marko Divéky, Peter Jurnečka, \\ Rudolf Kajan and L'uboš Omelina \\ Institution of Informatics and Software Engineering, Faculty of Informatics \\ and Information Technologies, Slovak University of Technology, \\ Ilkovičova 3,84216 Bratislava, Slovakia, bielik@fiit.stuba.sk, \\ WWW home page: http://www.fiit.stuba.sk/ bielik
}

\begin{abstract}
In this paper, we describe an innovative concept of threedimensional interactive educational games that combine the excitement and looks of popular computer games with the educational potential of electronic study materials and its realization by a system called S.M.I.L.E. - Smart Multipurpose Interactive Learning Environment. One of its key features is the consideration of various learners' abilities. In this paper, we concentrate on the description of features that enable different users (including handicapped) to learn effectively by playing educational games. We follow the idea that everyone needs access to quality education and are convinced that by enabling cooperative education not just among learners, but also between handicapped and able-bodied ones, we bring the humane dimension into education.
\end{abstract}

\section{Introduction}

Education has significantly evolved for the last decade, thanks to modern information technologies. Learners not only have access to a countless number of diverse educational materials, but also can educate interactively and share their ideas and knowledge with each other - even if they are miles apart. However, only a few of them are adaptive and therefore provide better support for individual learners [1].

Many learners lack motivation into studying. Educational games (i.e. serious games) are a new element that evolves rapidly [2]. Computer games not only offer enjoyment and fun, both of which play a crucial role in effective learning [3], but also do a great job in motivating. However, the most popular ones are currently far away from being educational and, on the other hand, educational games often lack the thrill of their popular counterparts and have a fixed plot that cannot be altered.

Another problem that needs to be addressed is the fact that learners with a handicap are often ignored and forgotten. Handicapped learners have absolutely no options to play today's modern educational computer games. We propose an 
innovative concept that forms a unique solution for the above mentioned problems. It combines the advantages from both interactive educational materials and popular computer games by giving teachers (i.e. authors of educational materials) the ability to transform study materials into exciting educational games that can be played even by handicapped (e.g., visually impaired or deaf) learners.

\section{Educational Games Model and its Realization}

Our model of educational games is based on today's most popular genre of computer games - Role Playing Games (RPGs). We focused on three-dimensional games, since their two-dimensional counterparts have been overcome by today's modern games. Moreover, 3D games support the development and training of spatial memory, what is important for everyone, but especially for vision-impaired users.

Games based on the RPG genre take place in a realistic world set in a specific time or in an imaginary world that is or is not close to reality. Players are represented by and control an avatar and solve various quests throughout the game. These require the player to find certain objects that he needs to correctly use or combine in order to solve a particular problem, and/or require the player to choose a correct answer to a certain question. Quests are assigned to players by Non-player Characters (NPCs) that cannot be controlled by players and interact with them through dialogs.

Our educational games model consists of two environments that encourage learners to first train and educate themselves and afterwards to test their newly gained knowledge and skills. The learning environment intended for self-training and raising the players' level of knowledge, presents a persistent virtual world that is made of quests based on all available educational materials. It practically resembles one vast game that contains all possible educational quests.

The second environment consists of teacher-created games. It presents a practical and enjoyable form of evaluating the players' level of knowledge and skills from a desired field. Games are specified by teachers either visually (by defining their concept) or automatically generated according to parameters set by the teacher. The visual approach to game creation makes our solution innovative, since nearly all current games do not offer mechanisms for creating games and their environments suitable for teachers who are not IT professionals. However, it is the automatic game generation based on teachers' preferences that makes our approach distinctive. The automatic generation of educational games consists of two separate phases: the Quest Generation and Game Generation phases that utilize artificial intelligence-based algorithms for solving particular tasks (e.g., simulated annealing to find appropriate landmarks during the landscape generation process, or depth search strategy with backtracking for quest generation). This process is described in more detail in [4].

\section{Considering Different Learners}

Having handicapped learners in mind, we chose to provide interfaces for all, and thus we designed the educational games with the possibility to be fully controlled via 
voice recognition and with peripherals for the handicapped, such as Braille keyboards. A mobile phone's keypad or PDA can also be used as an input device.

Diversity in Vision. Games can be played by means of voice-activated commands using a version of the Game Client specialized for blind and visionimpaired users. To other players, a blind player looks just like any other player, with the exception that he moves according to waypoints automatically placed when the game is created. Players with minor vision impairment can customize the visual appearance of important in-game elements, such as NPCs.

Diversity in Hearing. The default used interface is purely visual, and so the design does not need to be altered in order to provide support for deaf learners. The only exception to this rule is the playback of videos in knowledge materials. Nevertheless, hearing-impaired learners can choose to have subtitles shown below all played videos. On the other hand, all created educational games utilize environmental and character sounds in their otherwise visual interfaces. Deaf players, and also players with a hearing impairment use interfaces that contain extended graphical effects instead of sounds of any kind. In case a sound should be played, such players are shown a graphical image associated to the particular sound.

Diversity in Levels of Knowledge. Thanks to the fact that we can estimate the level of knowledge of each subject for every player and the fact that each quest is classified according to subjects it is related to, all educational games are adapted individually to every player's (estimated) level of knowledge and skills. Players are dynamically navigated into solving quests (e.g., by pointing players at map locations of the in-game NPCs that assign them) most suitable for their estimated knowledge level. Additionally, while solving quests, options of the appropriate difficulty are chosen for players to solve.

By utilizing the Item Response Theory [5], we are able to predict how will players react to both tasks and questions they are given while solving quests, and thus to measure their level of forgetfulness by testing them on tasks and questions they have previously successfully completed and answered.

Diversity in Skills. We let each and every player choose how he interacts with the educational games. For example, players who wish to interact with a standard keyboard have the option to specify their own controls. Naturally, players can also choose from a number of predefined control schemes and are able to further customize these schemes according to their liking.

Diversity in Styles. In the virtual world, every player is represented by an avatar - a virtual character according to which other players notice his presence. The option to personalize every player's avatar plays a crucial role in getting the players immersed into the gameplay and accepting the avatar as the player's own in-game identity. A player chooses a specific geometry model that can be further customized, e.g. players can specify its height by scaling the desired model.

Naturally, creating and importing a custom geometry model is rather difficult and cumbersome. Therefore, we have enabled players to assign different textures to various parts of their avatar's model, what proved as an effective and amusing method in differentiating players' avatars. Each geometry model, representing the body of an avatar, has distinct zones representing various body parts, e.g. a head, torso, hands and feet and players have the option to assign a desired texture (clothing) to each zone. A great advantage of this approach is the fact that the 
number of possible different avatars created grows exponentially according to the number of available geometry models and textures.

Additionally, a unique feature of our S.M.I.L.E. system is the possibility of players to give their avatars their own faces via textures created from the players' photographs (specified in a special format that allows automatic mapping of significant face elements). This feature is especially important and useful for educational purposes (as in opposite it may not be desirable in many cases for standard computer games where players usually do not want to present their identity).

\section{Conclusions}

The main contribution of the presented work is in devising a novel concept of threedimensional educational computer games that can be adapted to different learners and to various abilities of a particular learner. A unique feature is the proposal of methods that allow teachers themselves to define educational games based on the stored educational materials without requiring any knowledge of programming. Three-dimensional educational games (including the persistent virtual world) are dynamically generated based on teachers' preferences. User interfaces are designed as adaptable in such a way that they support handicapped learners.

In order to validate the presented concept, we have developed a system called S.M.I.L.E. that encapsulates and combines the advantages of two distinct worlds interactive educational materials and popular computer games. It also enables handicapped learners to educate together with their non-disabled colleagues. Such concept has not yet been realized to our knowledge in any of the existing applications. We have had positive feedback on the adaptability and adaptivity features from both teachers and students at elementary and secondary schools for handicapped children, where we demonstrated the S.M.I.L.E. system.

Acknowledgement. This work was partially supported by the Cultural and Educational Grant Agency of the Slovak Republic, grant No. KEGA 3/5187/07.

\section{References}

1. P. Brusilovsky and C. Peylo, Adaptive and intelligent Web-based educational systems, Int. Journal of Artificial Int. in Education 13(2-4), 59-172 (2003).

2. M. Zyda, Creating a Science of Games, Comm. of the ACM, 50(7) 27-29 (2007).

3. G. Dryden and J. Vos, The Learning Revolution (Jalmar Press, Austin, 1999).

4. M. Divéky, P. Jurnečka, R. Kajan, L. Omelina, and M. Bieliková, Adaptive Educational Gameplay within Smart Multipurpose Interactive Learning Environment, in: Semantic Media Adaptation and Personalization, edited by P. Mylonas, M. Wallace, M. Angelides (IEEE CS, Los Alamitos, CA, USA, 2007), pp. 165-170.

5. F. Baker, The Basics of Item Response Theory, ERIC Clearinghouse on Assessment and Evaluation (University of Maryland, College Park, MD, 2001). 